\title{
Explorations of Applying Cloud Computing Technology to the Digital Library
}

\author{
Guangcheng Zheng \\ College of Software and Service Outsourcing, Suzhou Chien-Shiung Institute of Technology, \\ Jiangsu Taicang, 215411, China
}

Keywords: Digital library, Cloud computing technology, Application, Explore.

\begin{abstract}
In the 21st century of science and technology, the cloud computing technology has already been full applied in all industries, which has quite obvious effects. Nowadays, all libraries have realized the digitalization. To become popular and obtain development, the digital library needs to introduce the cloud computing technology. The major argument of this paper is to explore the application of the cloud computing technology to the digital library. By comparative analysis, it finally concludes that the cloud computing technology is worthy of being promoted in digital libraries.
\end{abstract}

\section{Introduction}

In the future, it is the trend of era development to apply the cloud computing technology to the digital libraries, which has generated huge impact on the digital libraries and made contributions to the development of Chinese digital libraries, no matter from the management or service level.

\section{Fundamental Cognition of the Cloud Computing Technology}

\section{Concept of the Cloud Computing Technology}

Clouding computing refers to that, the internet automatically splits the huge computing procedure into numerous small subprograms, and then transmits the results to user after the search and analysis by the huge system consisted of several servers. With the help of this technology, online service supplier can handle tens of millions of information within several seconds, so as to offer the same efficient online service as strong as "supercomputer".

\section{Technologies Included in the Cloud Computing}

The simplest cloud computing technology includes SE and online mailbox. Users can get a lot of related information by inputting simple instructions. Complicated cloud computing technologies, such as mobile phone and GPS, can develop more application services by the cloud computing technology.

\section{Importance of Applying the Cloud Computing Technology to the Digital Libraries}

\section{Extending the Service Scope}

The appearance of digital libraries has broken through the mode of traditional library, which has extended the functions of traditional libraries. With the consistent development of science and technology, people have got higher and higher requirements about the level of informatization. Under such a circumstance, people have to make corresponding innovations of the digital library technology. 
The cloud computing technology can improve the technologies of digital libraries, extend the services of digital libraries, satisfy people's requirements about the level of informatization, meet the demands of all different customers and improve the service level of digital libraries ${ }^{[1]}$.

\section{More Close Relationship with Customers}

The $21^{\text {st }}$ century is the era of network. Digital libraries and customers should communicate with each other by the network. Customers should be much closer to the information resource. Application of the cloud computing technology can meet the demand. Such as setting up QQ and WeChat, customers can consult various problems online and solve related problems for customers.

\section{Improving the Training and Evaluation Mechanism of College Libraries}

College libraries have played a crucial role in the society. Therefore, during the process of development and construction, it is the key to train and evaluate the management personnel. Currently, Chinese college libraries have not well managed the personnel and lacked corresponding management technology. To introduce the cloud computing technology, it can help the digital libraries to improve the management level, develop and improve the management of personnel, further to promote the construction of training and evaluation mechanism of digital libraries ${ }^{[2]}$.

All in all, the application of the cloud computing technology cannot only meet the demand of modern internet, but also meet different requirements of customers. It has broken through the fixed mode of traditional libraries and shown the unique charm of digital libraries. In the aspect of personalized selection, it is more convenient and rapid, which helps the digital libraries to realize the informatization of numbers and fatherly improve the construction of digital libraries.

\section{Introduction to the Cloud Computing in Digital Libraries}

\section{Basic Theory of Cloud Computing}

Related theories of the cloud computing are quite complicated. Many countries and scholars have made many researches on the cloud computing. However, due to the complexity of the cloud computing technology, there are many divergences during the researching process. In view of the current research results, people have not reached a consensus. However, scholars of the academic circle all agree that, the cloud computing is one kind of online and distributed computing. By integrating these basic opinions, people can form the basic theory of the cloud computing technology, so as to make better utilization of the cloud computing, make efficient integration of resources and transmit that to customers by internet, further to offer better services for customers.

\section{Connotations of the Cloud Computing}

From the characteristics of the cloud computing, it has many roles, such as convenient, fast, safe and reliable, sharing resources, virtualized service and economically environment-friendly. The largest advantage of the cloud computing lies in the integration of resources, customers can find corresponding resource and information quickly with the help of cloud computing technology. Besides, it is convenient and rapid. The biggest core idea of the cloud computing technology is that, a lot of resources are arranged and summarized by internet. All these information is under the same control, so as to form a resource pool with more improved service. Characteristics of the cloud computing technology fully show the uniqueness of the cloud computing technology in current era, which is worthy of being promoted and applied.

\section{Application of the Cloud Computing Technology}

The cloud computing technology has been widely applied throughout the world. Many countries have attached much importance to the application and development of the cloud computing technology, which does not only have the commercial values, but also social values to a certain extent. In the meanwhile of realizing the maximized economic profits, it is convenient for people to meet the 
demand of information resources ${ }^{[3]}$. Especially in recent years, digital libraries have vigorously promoted the cloud computing technology. Many foreign digital libraries have already applied the cloud computing technology. Moreover, they have made investigations and analysis on the application of the cloud computing technology to the digital libraries, further to better apply the cloud computing technology during constant application and development. Currently, in view of Chinese application of the cloud computing technology, the application of digital libraries has still been on the initial stage. However, in view of Chinese economic development, the cloud computing technology will be greatly applied in the digital libraries in future, which is not only the requirement of the global economization and development of the era, but also the requirement for China to realize the modern digital libraries.

\section{Concrete Application of the Cloud Computing Technology to Libraries}

\section{Reducing the Construction Cost of Libraries}

Compared to the traditional network mode, the cloud computing technology has good performance, which can reduce the disadvantage of server's restricted access to a certain extent. Servers barely have breakdowns, which can achieve the standard that the normal server cannot do. Under normal circumstance, overload information of normal server can result in breakdown, which increases the cost input. By comparison, the cloud computing technology can save more costs. People can get more cloud computing services by pay a little. In addition, it is fast. Customers are more satisfied with this service level, which can both save the costs and increase profits for the libraries. Such effects are just required by the current situation, to develop thrift economy and save costs.

To a certain extent, application of the cloud computing to the digital libraries has saved the cost of maintenance. In the aspect of information management, traditional library mode is featured by outdated idea and aging facility. It needs a lot of expenditure to maintain related facilities. Library is one public welfare project. The resource of fund mainly relies on the governmental finance. To a certain extent, the capital is quite tight. There are only few fees for maintenance, which results in old facilities of libraries. There are no corresponding funds to update facilities. The service level is quite slow. Introduction of the cloud computing technology can solve the problem, which has no limitations for the client. In this way, it can better promote the construction of terminal to visit the digital libraries and improve the service quality of libraries.

\section{Improving the Service Level of Libraries, Realizing the Integrated Resource Sharing Platform}

With the consistent development and progress of times, people have more requirements about the information in this era of informatization. Amount of information should reach a certain extent. Besides, the service quality should also satisfy the demands of the public. With the development of times, customers' demands of information have also developed. In the modern society, customers have had increasingly more requirements about the information resources, with an obviously rising tendency ${ }^{[4]}$. The traditional library service mode has no longer satisfied the demand of modern social development, or meet related requirements of users. It is urgent to change the traditional mode.

To realize the mode of integrated information and resources, the digital libraries can meet the requirements of users and catch up with the tide of the times development. Currently, it is the most reasonable and scientific way to set up the mode of information sharing by the application of the cloud computing technology. Related data shows that, China annually spends tens of thousands yuan on the sharing of data. However, it still cannot meet the demands of users to a certain extent, which cannot realize the complete sharing of information resources in a real sense. The cloud computing establishes the integrated resource sharing, form the unified database and set up the center of "cloud storage", further to achieve the resource sharing in a real sense and offer first-class technological services for users. For small and medium-sized libraries, it means more to them by setting up integrated information resources. Due to the small development scale of themselves, there are a small variety of books. Through the cloud computing technology, it can realize resource sharing. All 
resources in the library industry can be shared, which helps the small and medium-sized libraries to walk out of the dilemma, and also give the full play to the social effects of digital libraries.

\section{Guaranteeing the Normal Operation of Digital Libraries}

In view of the current book collection in China, there is a lot of information, which needs advanced server to guarantee the normal circulation of information. The cloud computing technology happens to solve the problem. While undertaking a lot of information resources, the serving system will not break down, but work normally, which will not result in the standstill of digital library's system.

Due to the deficiencies of traditional library's management mode, many books are damaged and lost, thus information resources cannot be completely presented. In addition, it cannot guarantee the security of data. The management of system lacks advanced technologies. The system is often infected by virus. Besides, the phenomena of delay often occur. Loss of data will result in incomplete information resource and let the digital library to suffer the damage of reputation. To a certain extent, the application of cloud computing technology can guarantee the security of information data and normal operation of the system, which will not bring inconvenience to users. No matter the technology or role, the cloud computing technology is the best choice for the digital library.

\section{Storing and Retrieving Information by the Cloud Computing}

During the development process of digital libraries, the biggest problem is the storage of information, which can be resolved by the cloud computing technology. During the construction of cloud computing, it can store the information resources of libraries by the distributed storage. With the help of several servers, it can make distributed storage of various information. Meanwhile, it can store the information resources, so as to avoid the breakdown of system and loss of information.

The cloud computing technology can also store information of users, including the basic information of user's operation. Such as the contents and times, it all makes statistics and storage, arrange ans sequence the information of users. Moreover, the security of information stored by the cloud computing is quite high. Professional team arranges and summarizes the relevant resource information, enhance the security of data sharing and protect the data to a certain extent. Data storage and retrieval by the cloud computing can be guaranteed, which offers conditions for the digital libraries to realize the networking.

\section{Offering the Platform for Supporting the Public Service}

The cloud computing offers technological support for the establishment of digital libraries and optimizes the service level of digital libraries, which improves the libraries' management effects of books. It makes the theoretical integration of resource information and improves the construction level of digital libraries. The cloud computing technology has promoted the realization of digital libraries' networking progress. Relying on better public service supporting platform, digital libraries can develop better.

\section{Problems to be Noticed by the Cloud Computing Technology in Digital Libraries}

\section{Implementing the Application of Cloud Computing Selectively}

While managing the information of books, it should select the cloud computing technology in real time. Before implementing the cloud technology, it needs to consider the actual situations of the library and reasonably apply the cloud computing technology step by step.

\section{Selecting Highly-valued Suppliers}

While applying the cloud computing technology, it needs to pay attention to select suppliers which are highly valued. Whether they can offer customized cloud service, transplant and dig a large amount of data and expand the corresponding cloud computing service, all these can be the criterion for selecting suppliers. 


\section{Avoiding the Interference of Intellectual Property}

While applying the cloud computing technology, it also needs to consider the related property problem. Libraries need to formulate a set of related program and avoid the occurrence of intellectual property disputes.

\section{Simplifying Business and Adjusting Structure}

According to corresponding cloud computing technology, digital libraries need to make business more refine and simplified, make corresponding adjustments of the structure, so as to catch up with the development of cloud computing. Relevant management and responsible officers should gradually virtulize and decompose the business, so as to let the digital libraries to organically integrate the cloud computing mode and current development mode level ${ }^{[5]}$.

\section{Conclusion}

The cloud computing technology has been increasingly applied to the global digital libraries. Therefore, it should arouse the attention of China. Currently, China has still been on the initial stage of applying the cloud computing technology to digital libraries, which needs to pay attention to corresponding technological problems during the application, positively solve the existing problems, lay a good foundation and make preparations for Chinese digital libraries to fully apply the cloud computing technology, offer technical support for the construction of sharing platform integrated of resources and information, and also make contributions to the construction of first-class digital library in China.

\section{Acknowledgement}

This paper is the Youth Fund Project of Ministry of Education (15YJC880143).

\section{References}

[1] Shen Jie. Brief Analysis on the Application of Cloud Computing Technology to the Construction of Digital Library Platform, Science and Technology Vision, 2014,36:271+337.

[2] Tang Jinxiu. Researches on the Application of Cloud Computing Technology to the Digital Library, Lantai World, 2013,23:132-133.

[3] Wang Bin, Wang Yuhang. Explorations of Application of Cloud Computing Technology to the Digital Library, Wireless Internet Technology, 2015,24:21-22.

[4] Qin Jiuying. Explorations of Applying the Cloud Computing Technology to the Digital Library, Examination Weekly, 2015,78:114.

[5] Zong Hongwei. Explorations of Applying the Cloud Storage Technology to the Construction of Digital Library, Science and Technology Information Development and Economy, 2014,17:130-132. 